\title{
Prostate Lymphoscintigraphy and Radio-Guided Surgery for Sentinel Lymph Node Identification in Prostate Cancer
}

\author{
Technique and Results of the First 350 Cases
}

\author{
Friedhelm Wawroschek ${ }^{a}$ Harry Vogt $^{b}$ Hermann Wengenmair ${ }^{b}$ \\ Dorothea Weckermann ${ }^{a}$ Michael Hamma Mathias Keila Gerhard Graf ${ }^{b}$ \\ Peter Heidenreich ${ }^{b}$ Rolf Harzmann ${ }^{a}$
}

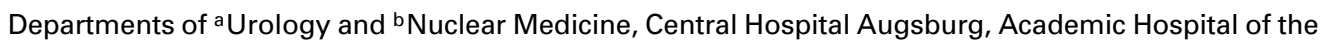

Ludwig Maximilian University Munich, Augsburg, Germany

\section{Key Words}

Prostatic neoplasms · Lymph node excision •

Radionuclide imaging · Lymphoscintigraphy · Sentinel lymph node

\begin{abstract}
Introduction: Having in mind the promising results of lymphoscintigraphy and intraoperative gamma probe application for the detection of sentinel lymph nodes (SLN) in malignant melanoma, breast and penis cancer, we tried to identify the SLN in prostate cancer by applying a comparable technique. Materials and Method: 350 patients with prostate cancer were examined after providing informed consent. The day before pelvic lymphadenectomy technetium-99m nanocolloid was transrectally injected into the prostate under ultrasound guidance. A single central application was done per prostate lobe in most cases. Activity attained 90$400 \mathrm{MBq}$, and the total injected volume was about 2-3 $\mathrm{ml}$. Hereafter, lymphoscintigraphy was carried out. Those lymph nodes having been identified as SLN by means of gamma probe detection and lymphoscintigraphy were removed intraoperatively. Later, most of the
\end{abstract}

\section{KARGER}

Fax +41613061234

E-Mail karger@karger.ch

www.karger.com
(C) 2003 S. Karger AG, Basel

0042-1138/03/0704-0303\$19.50/0

Accessible online at:

www. karger.com/uin cases had different types of pelvic lymphadenectomy. SLN received serial sections and immunohistochemistry, non-SLN step sections. Results: 335 patients showed at least $1 \mathrm{SLN}$ in lymphoscintigraphy. $24.7 \%$ had lymph node metastases. In 2 patients, metastases in non-SLN were found without at least one SLN being affected (false-negative patient). Conclusion: Our experience suggests that the SLN identification is not only feasible in breast cancer and malignant melanoma, but also in prostate cancer with a comparable technique.

Copyright $\odot 2003$ S. Karger AG, Basel

\section{Introduction}

The first papers about lymphoscintography of the human prostate were published in the late 1970s and early 1980s [1-4]. Previous investigations had the objective to prove the presence of an intraprostatic lymphatic system and the imaging of regional lymphatic drainage from the prostate. Gardiner et al. [1] were the first to demonstrate lymphatic pathways by transrectal injection with a 99mTc-labelled tracer (particle size 4-12 $\mathrm{nm}$ ) into the prostate capsule. Transperineal injection into the parenchyma 
Fig. 1. Typical prostate lymphoscintigraphy with early (30 min) and delayed ( $3 \mathrm{~h})$ imaging. Multiple sentinel (black arrows) and subordinated lymph nodes.

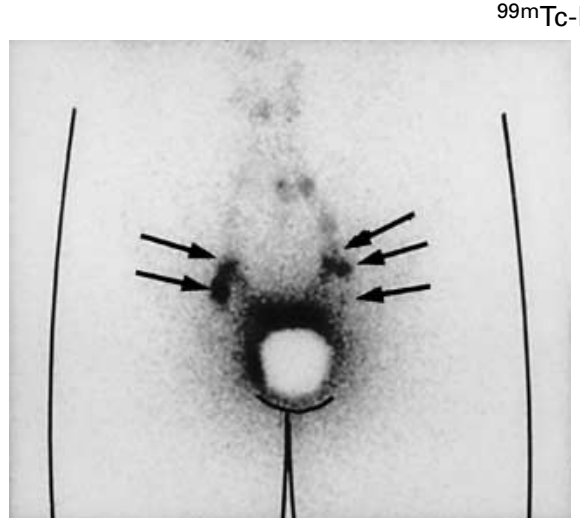

RVL 30 min

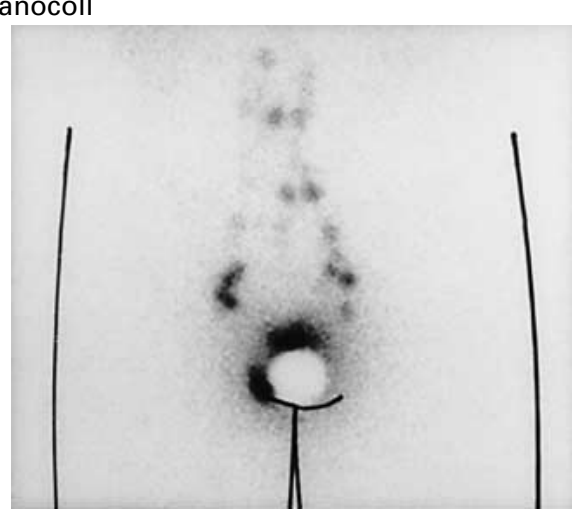

RVL 3 h

99mTc-Nanocoll

March 08, 1999

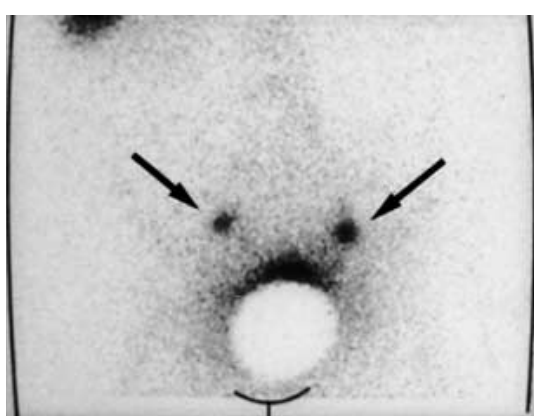

RVL 20 min

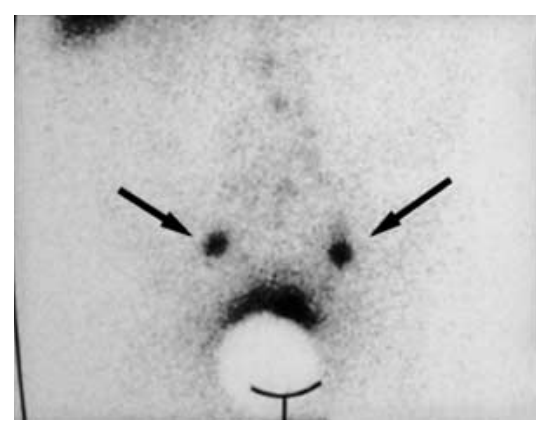

RVL $2 \mathrm{H}$
March 25, 1999

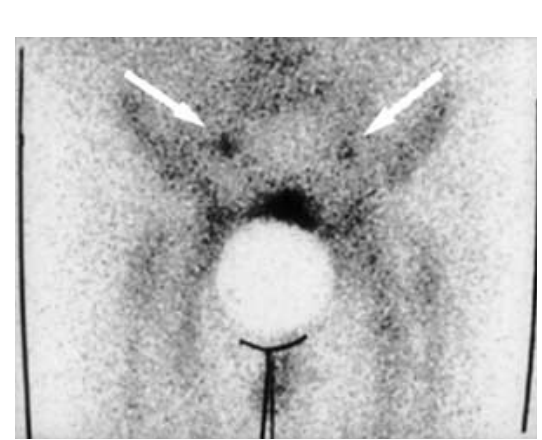

RVL 20 min

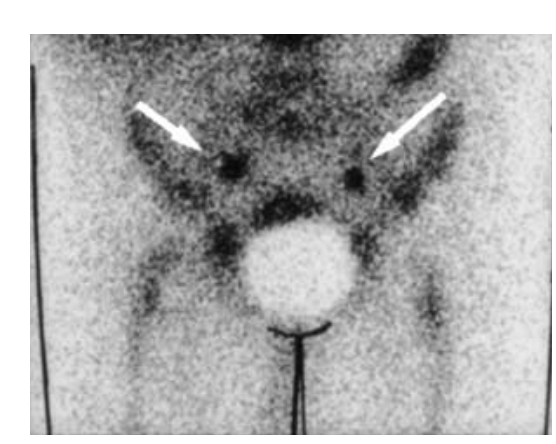

RVL $2 \mathrm{H}$

Fig. 2. A repeated prostate lymphoscintigraphy (the day of surgery had to be postponed for unforeseen reasons) with identical SLN location (black and white arrow).

as well as open and periprostatic injection were not successful. In all 40 patients with injections close to the capsule a uni- or bilateral lymph drainage was shown. In 1990, Zuckier et al. [5] were able to show partly contralateral, pelvic lymphatic drainage in 8 of 9 patients with prostate cancer using a comparable technique. A single patient needed a repeated injection under similar conditions and showed an identical lymphatic drainage. Whether it was possible to depict the entire lymphatic drainage of the prostate remains unclear, because operative and histological reviews on patients were not carried out. 
After the intended verification of metastases by prostate lymphoscintography failed, it was not developed any further in the 1990s. Only after the clinically important combination of preoperative lymphoscintigraphy and intraoperative gamma-probe measuring took place, similar to the procedure in breast cancer and malignant melanoma, did the method experience a revival $[6,7]$.

\section{Patients and Method}

\section{Patients}

Between July 1998 and October 2001, 350 patients with prostate cancer were examined after providing informed consent. Special criteria for the selection of the patient population were not applied. Preoperative total body bone scanning of the pelvis showed normal findings in all patients. The average preoperative PSA level was $12.7 \mathrm{ng} / \mathrm{ml}$.

\section{Tracer Injection}

The day before pelvic lymphadenectomy technetium- $99 \mathrm{~m}$ nanocolloid (Nanocoll ${ }^{\circledR}$, Sorin Co., Italy) was applied transrectally into the prostate under ultrasound guidance. The interval between injection and surgery varied between 18 and $22 \mathrm{~h}$. A 1-day protocol was an exception. A single central application was done per prostate lobe, rarely two. Depending on the interval between injection and surgery the chosen activity attained $90-400 \mathrm{MBq}$ (median 267.3 MBq) and the total injected volume was about 2-3 ml.

\section{Prostate Lymphoscintigraphy}

In the beginning, approximately $30 \mathrm{~min}$ later and a few hours after injection, scintigraphy (Sopha camera, DSX, Leap collimator) was performed in anteroposterior projections (fig. 1,2), and in single case lateral views. The prostate was, in part, covered during this procedure. Later the taking of early images was abandoned, because they did not supply additional information about SLN identification. Initially, the SLN were noticed to be covered by the inevitable overflow of radioactivity into the urine of the bladder, especially in the early images. This problem was reduced by transurethral catheterization. After early imaging was abandoned this measure was unnecessary.

\section{Operative Procedure}

In relation to the intended therapy of prostate cancer (e.g. radiotherapy, perineal or retropubic prostatectomy), pelvic lymphadenectomy was carried out via different surgical accesses (table 1). In the majority of patients a lymphadenectomy was directly followed by radical retropubic prostatectomy $(\mathrm{n}=282)$. In the beginning, all patients received sentinel lymphadenectomy (SLNE) plus the dissection of the lymph nodes from the obturator fossa and the external iliac lymph nodes (so-called modified lymphadenectomy). Analysis after 121 patients [7] showed that sentinel lymphadenectomy alone in comparison to modified lymphadenectomy has a sensitivity of about $96 \%$. This result was statistically significant $(\mathrm{p}<0.05)$ compared to resection of the obturator lymph nodes only. Subsequently, we modified our study protocol. In the following, the extent of pelvic lymphadenectomy varied depending on the preoperative risk factors between SLNE only (PSA $<10 \mathrm{ng} / \mathrm{ml}$, Gleason Score $<7$ in biopsy and clinical stage $<$ T3) and SLNE with additional extended pelvic
Table 1. Different surgical approaches of pelvic lymphadenectomy

\begin{tabular}{lc}
\hline Surgical approach & Cases \\
\hline Laparotomy & 272 \\
Minilaparotomy & 69 \\
Laparoscopy & 7 \\
\hline Total & 348 \\
\hline
\end{tabular}

Table 2. Extension of pelvic lymphadenectomy

\begin{tabular}{lc}
\hline Extension of pelvic lymphadenectomy & Cases \\
\hline $\begin{array}{l}\text { Sentinel lymphadenectomy } \\
\text { Sentinel lymphadenectomy plus modified pelvic }\end{array}$ & 129 \\
$\quad$ lymphadenectomy & 121 \\
$\begin{array}{l}\text { Sentinel lymphadenectomy plus extended } \\
\quad \text { lymphadenectomy }\end{array}$ & 94 \\
Pick-up lymphadenectomy & 4 \\
\hline Total & 348 \\
\hline
\end{tabular}

lymphadenectomy (PSA $>10 \mathrm{ng} / \mathrm{ml}$, Gleason Score $>6$ in biopsy or clinical stage $>$ T2) (table 2). Two cases received no operation after lymphoscintigraphy.

The radioactivity of the lymph nodes was measured intraoperatively by different gamma probes (C-TRAK system, Car-Wise, Morgan Hill, Calif., USA and Szintiprobe MR-100, pol. hi. tech., Italy). The identification technique was similar to that used in other tumor entities.

\section{Results}

\section{Patients and Lymph Node Histology}

Allergic or septic complications did not occur during perioperative antibiotic prophylaxis with a chinolon derivate. In the absence of rectal disease (e.g. anal fissure) the injection was well tolerated.

86 of 348 patients $(24.7 \%)$ had lymph node metastases. SLN received serial sections (5 systematically distributed sections of $4 \mu \mathrm{m}$ between each step section) and immunohistochemistry (IHC, pancytokeratin antibody), non-SLN step sections of approximately $2 \mathrm{~mm}$. In 2 patients, lymph node metastases in non-SLN were shown without a single SLN being affected. The incidence of node-positive patients depends on preoperative prostate-specific antigen 


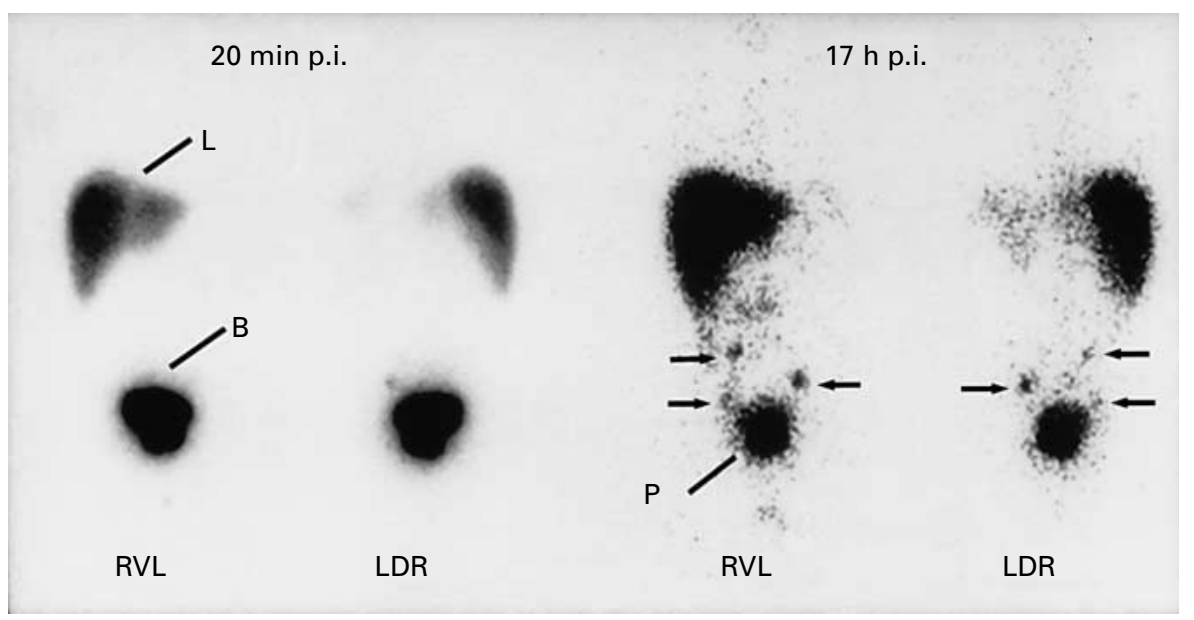

Fig. 3. Whole-body scan with distribution of radioactivity into the liver (L), bladder (B), prostate (P), and SLN (black arrows).

Table 3. Lymph node status depending on preoperative prostate specific antigen (PSA) level (a) and tumor classification of the prostatectomy specimen (b)

a

\begin{tabular}{lrc}
\hline PSA, ng/ml & Cases & Node-positive patients \\
\hline $0-4$ & 19 & 2 \\
$>4-10$ & 168 & 29 \\
$>10-20$ & 113 & 33 \\
$>20$ & 50 & 22 \\
\hline Sum & 350 & 86 \\
\hline
\end{tabular}

b

\begin{tabular}{lrl}
\hline Tumor classification & Cases & Node-positive patients \\
\hline T1,2a,b & 171 & 26 \\
T3a & 55 & 15 \\
T3b & 40 & 28 \\
T4 & 16 & 11 \\
\hline Sum & 282 & 80 \\
\hline
\end{tabular}

(PSA) and tumor classification of the prostatatectomy specimen (table 3a, b).

$69.8 \%$ of the node-positive cases had no positive nonSLN and approximately 2 of 3 had only one positive SLN.

\section{Prostate Lymphoscintigraphy}

In $92.6 \%$ of the patients at least a single SLN was revealed in lymphoscintigraphy. Prostate lymphoscintigraphy seemed only to be influenced by a neoadjuvant hormonal therapy provided it was the selected treatment over a longer period of time ( $>3$ months). In 10 randomly selected patients the determination of radionuclide distribution (fig. 3) and its time course was made via 'Regions of Interest' (ROIs) over prostate, bladder, liver, spleen and the SLN [8] (table 4). This revealed a high interindividual variance of uptake.

The depiction of lymphatic pathways was only possible in $11.1 \%$ of the cases. The essential SLN criteria were the persisting accumulation of activity and the anatomical localization.

\section{Intraoperative SLN Identification}

In 10 patients it was not possible to identify at least one SLN (2.9\%) intraoperatively: one patient received neoadjuvant hormonal therapy for 3 years, 2 had laparoscopic surgery, and 3 had transurethral or transvesical prostate surgery for benign enlargement before. In 4 cases no reason was obvious. In an additional 5 patients, the intraoperative measuring was not applied (e.g. local advanced disease) or not completed (e.g. macrometastases). Of 23 patients who had a previous transurethral resection or transvesical prostatectomy 4 showed no SLN. The limitation was significant $\left(\alpha<0.005, \chi^{2}\right.$ test $)$.

We dissected an average of 5.6 SLN and 12.4 nonSLN. Of those patients with at least one SLN, 78.9\% had between 2 and 8 SLN (fig. 4). There was no significant 


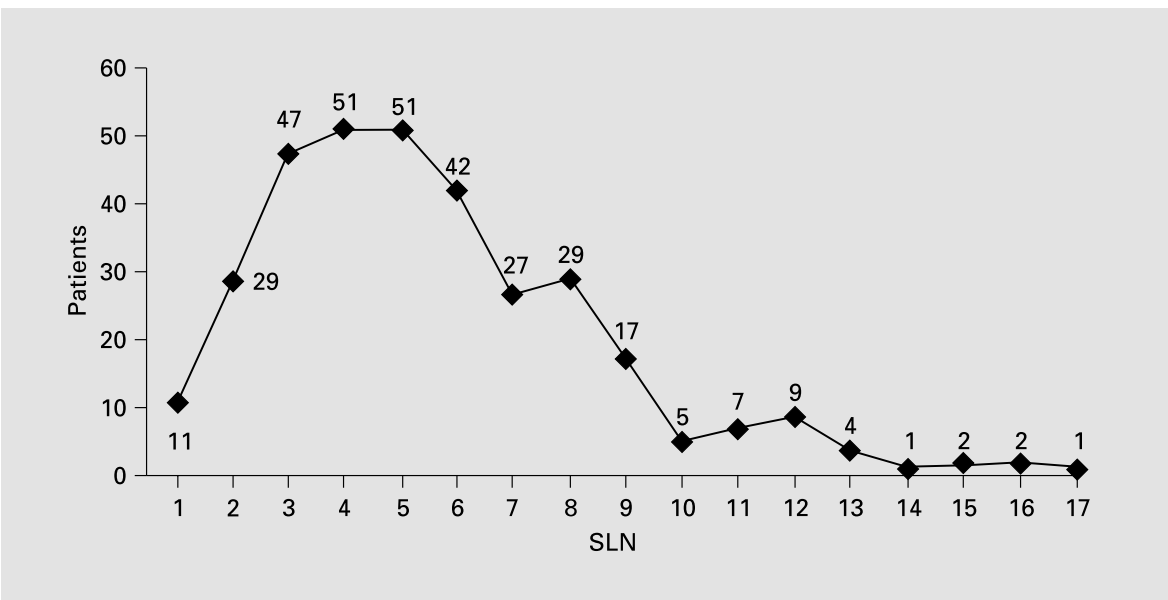

Fig. 4. Number of SLN in each patient.

(Mann-Whitney test) dependence on the number of SLN, tumor stage, and number of SLN in node-positive and -negative patients (5.8 vs. $5.5 \mathrm{SLN}$ ).

All radioactive lymph nodes which could not be clearly identified as a subordinated lymph node on the basis of the anatomical localization and knowledge of the lymphoscintigraphy were classed as SLN. In 78.6\%, the number of intraoperatively identified SLN exceeded the ones determined by scintigraphy. In $16.4 \%$ of the cases dissection of all SLN was impossible. Mostly, this was the case in laparoscopy (6 of 7 cases) or minilaparotomy (16 of 69 cases). Non-resectable or reidentifiable SLN were mostly located in areas which were difficult to access surgically (internal iliac region, pararectal, presacral).

\section{Discussion}

Lymph node metastases are relatively often revealed in supposedly localized prostate cancers at the time of surgery. Data for the frequency of this manifestation differ largely depending on the treated population and the extent and technique of pelvic staging lymphadenectomy.

While large US studies revealed lymph node-positive stages in only $12 \%$ [9], we detected lymph node metastases in nearly $24.7 \%$ in our own investigation, despite inconspicuous preoperative imaging (CT, MRI).

The lymph node status in prostate cancer is not only of prognostic but also of tremendous therapeutic relevance, since common standards still include the renunciation of local curative therapy (e.g. radical prostatectomy, radiotherapy) in case of positive lymph node findings and pos-
Table 4. Average uptake (\%) of different organs in prostate lymphoscintigraphy

\begin{tabular}{lc}
\hline Organ & $\begin{array}{l}\text { Average uptake } \\
\% \text { (range) }\end{array}$ \\
\hline Prostate & $15.8(7.7-28)$ \\
Bladder & $24.5(4.3-47)$ \\
Liver & $25(12.2-47.2)$ \\
Spleen & $2.1(0.7-3.6)$ \\
Bone marrow & $32(21.9-54.2)$ \\
Sentinel lymph nodes & $0.12(0.007-0.63)$
\end{tabular}

tulate hormonal withdrawal [10]. Thus, all techniques for the identification of metastases should have a high sensitivity (in order to avoid unnecessary and stressful treatment) and a high specificity (in order not to deprive a patient of a promising curative treatment).

Pelvic lymphadenectomy is presently considered the method of choice for the identification of lymph node metastases in prostate cancer [11]. Alternative techniques for a sufficient prediction of lymph node micrometastases have not been developed. Pelvic CT scans and MRI do not give certainty on the presence of lymph node micrometastasis [12]. Even radioimmunoscintigraphy with indium-111-capromab pendetide is presently not able to replace pelvic lymphadenectomy, showing a positive predictive value for a high-risk collective of only $66.7 \%$ [13]. This is also the case for the application of positron emission tomography with fluorine-18 fluorodeoxyglucose, in which a large number of micrometastases remain undetected $[14,15]$. 
Table 5. Published complication rates of pelvic lymphadenectomy

\begin{tabular}{lcll}
\hline Author & Patients & $\begin{array}{l}\text { Complication } \\
\text { rate, \% }\end{array}$ & $\begin{array}{l}\text { Type of } \\
\text { lymphadenectomy }\end{array}$ \\
\hline Paul et al. [16], 1983 & 150 & 51 & extended open \\
Mc. Dowell et al. [18], 1990 & 217 & 22 & extended open \\
Schuessler et al. [17], 1993 & 147 & 31 & extended laparoscopic \\
Stone et al. [26], 1997 & 150 & 35.9 & extended laparoscopic \\
& 39 & 2 & modified laparoscopic \\
Lezin et al. [27], 1997 & 22 & 9.1 & modified minilaparotomy \\
& 22 & 31.8 & modified laparoscopic \\
modified laparoscopic \\
Fahlenkamp et al. [28], 1997 & 200 & 12.5 & modified open \\
Herrell et al. [29], 1997 & 38 & 20 &
\end{tabular}

Table 6. Incidence of node-positive patients depending on preoperative risk factors

\begin{tabular}{lccc}
\hline Preoperative risk factors & \multirow{2}{*}{ Patients } & \multicolumn{2}{c}{ Node-positive patients } \\
\cline { 3 - 4 } & & $\mathrm{n}$ & $\%$ \\
\hline PSA $<10 \mathrm{ng} / \mathrm{ml}$ and $<\mathrm{cT} 3$ and Gleason score in biopsy $<7$ & 153 & 18 & 11.8 \\
PSA $>10 \mathrm{ng} / \mathrm{ml}$ or $>$ cT2 or Gleason score in biopsy $>6$ & 197 & 68 & 34.5 \\
\hline
\end{tabular}

Homogeneous surgical standards of pelvic staging lymphadenectomy for prostate cancer cannot be gathered from the current literature. Because of the high morbidity due to extended pelvic lymphadenectomy (table 5) and the operating time of an average $2.5 \mathrm{~h}$ [16] the area of dissection has been decreased at most centers. Published data of extended pelvic lymphadenectomy in prostate cancer $[17,18,19]$ point out that any limitation of the dissection area corresponds with a reduced detection rate of micrometastases. The widespread limitation of the dissection area to the so-called obturator fossa lymph nodes results in missing about $50 \%$ of the lymph-node-positive patients [17]. This and the at times surgically rather difficult accessible locations (presacral, pararectal, paravesical, hypogastric lymph nodes) of the primarily draining lymph nodes of the prostate explain the clinical need of radio guided lymph node surgery in prostate cancer.

The SLN identification in prostate cancer is different on principle from the techniques in other tumor entities. In breast cancer, penile cancer and malignant melanoma mostly a well-placed peritumoral injection is carried out, only to demonstrate the lymphatic drainage from the tumor. In prostate cancer, however, it is not known preoperatively which part of the organ the metastatic spread is originating from. Therefore, the principle aim has to be the identification and resection of all primarily draining lymph nodes, inevitably comprising a single or more SLNs of the tumor.

There are numerous explanations for the number of intraoperatively identified SLN exceeding the ones found preoperatively in lymphoscintigraphy.

(1) Following precise preparation, single hot spots proved to be several neighboring radioactive lymph nodes.

(2) Overlapping radioactivities of lymph nodes from different levels.

(3) Lymph nodes closely related to the prostate are covered by intraprostatic activity.

(4) Delayed lymphatic drainage following lymphoscintigraphy.

Furthermore, it is not possible to demonstrate for every single radioactive lymph node, whether it is a primarily draining lymph node or a subordinate. This is explained by the mostly absent lymphatic pathways and the particularly delayed lymphatic drainage. However, this problem is of minor clinical relevance, because only in $14.3 \%$ of the patients more than 8 SLN were identified. The average number and location of the identified SLN corresponds with anatomical descriptions of the prostatatic lymphatic drainage in humans. 
Our experiences with this novel technique point out the necessity of preoperative lymphoscintigraphy since the minimal number of SLN to be detected intraoperatively is determined by this method. Furthermore, it is easier to locate obscured and surgically difficult accessible lymph nodes intraoperatively. This is valid especially in minimal invasive surgery.

Our investigation demonstrates that the number of lymph-node-positive patients is considerably larger than mentioned in the current literature [9]. Even with comparatively favorable preoperative prognostic factors (PSA $<10 \mathrm{ng} / \mathrm{ml}$, clinical stage $<\mathrm{T} 3$, and Gleason Score $<7$ in prostate biopsy) we found lymph node metastases in $11.8 \%$ of the cases (table 6), although staging lymphadenectomy is no standard option. In addition, the individual variability of the lymphatic drainage and in case of limited dissection area the reduced sensitivity for detecting node-positive patients is obvious: approximately $60 \%$ would be missed with the customary pelvic lymphadenectomy limited to the lymph nodes in the obturator fossa (table 7).

In principle during the period of validation it has to be realized that to determine the sensitivity of a procedure a comparably extended lymphadenectomy has to be carried out. Additionally, all lymph nodes have to be examined with the same histopathological technique. As we investigated all sentinel lymph nodes in this study with IHC and serial sections, we consciously give no sensitivity. Taking
Table 7. Identified node-positive patients depending on the extension of pelvic lymphadenectomy (based on the results of sentinel lymphadenectomy, with or without additional pelvic lymphadenectomy and with serial sections and immunohistochemistry of all sentinel lymph nodes, non-SLN received step sections); $95 \%$ CI

\begin{tabular}{lc} 
Region of lymphadenectomy & $\begin{array}{c}\text { Node-po } \\
\text { patients, }\end{array}$ \\
\hline $\begin{array}{ll}\text { Obturator fossa, external and internal iliac region, } \\
\quad \text { presacral, pararectal, paravesical }\end{array}$ & 100 \\
Obturator fossa, external and internal iliac region & 96.5 \\
Obturator fossa, internal iliac region & $79^{*}$ \\
Obturator fossa, external iliac region & $75.6^{* *}$ \\
Obturator fossa & $40.7^{* *}$
\end{tabular}

* Significant for $\mathrm{p}<0.05$; ** significant for $\mathrm{p}<0.01$.

into account the above-mentioned criteria, the sensitivity of sentinel lymphadenectomy alone varied between 93.1 [30] and 96\% [7].

Improving the accuracy of the preoperative SLN identification (e.g. visualization of lymphatic pathways, decrease of SLN count rate, increase of urine contamination) based on the evaluation of different parameters (e.g. radiopharmaceutical, injection site, injection volume) must be the most important goal of further investigations.

\section{References}

1 Gardiner RA, Fitzpatrick JM, Constable AR, Crawage RW, O'Donoghue EPN, Wickham JEA: Improved techniques in radionuclide imaging of prostatic lymph nodes. Br J Urol 1979;51:561-564.

2 Kaplan WD, Whitmore WF, Gittes RF: Visualization of canine and human prostatic lymph nodes following intraprostatic injection of technetium $99 \mathrm{~m}$ antimony sulfide colloid. Invest Radiol 1980;15:34-38.

3 Stone AR, Merrick V, Chisholm GD: Prostatic lymphoscintigraphy. Br J Urol 1979;51:556560.

4 Whitmore WF, Blut RD, Kaplan WD, Gittes RF: Radiocolloid scintigraphic mapping of the lymphatic drainage of the prostate. J Urol 1980;124:62-67.
5 Zuckier LS, Finkelstein M, Kreutzer ER, Stone PL, Freed SZ, Bard RH, Blaufox MD, Freeman LM: Technetium-99m antimony sulphide colloid lymphoscintigraphy of the prostate by direct transrectal injection. Nucl Med Commun 1990;11:589-596.

6 Wawroschek F, Vogt H, Weckermann D, Wagner Th, Harzmann R: The sentinel lymph node concept in prostate cancer: First results of gamma probe guided sentinel lymph node identification. Eur Urol 1999;36:595-600.

7 Wawroschek F, Vogt H, Weckermann D, Wagner Th, Hamm M. Harzmann R: Radioisotope guided pelvic lymph node dissection for prostate cancer. J Urol 2001;166:17151719.

8 Wengenmair H, Kopp J, Vogt H, Wawroschek F, Gröber S, Dorn R, Heidenreich P: Sentinel lymph node diagnostic in prostate carcinoma. II. Biokinetics and dosimetry of ${ }^{99 \mathrm{~m} T c-n a n o-}$ colloid after intraprostatic injection. Nucl Med 2002;412:102-107.
9 Partin AW, Kattan MW, Subong ENP, Walsh PC, Wojno KJ, Oesterling JE, Scardino P, Pearson JD: Combination of prostate-specific antigen, clinical stage, and Gleason score to predict pathological stage of localized prostate cancer. JAMA 1997;277:1445-1451.

10 Aus G, Abbou CC, Pacik D, Schmid HP, van Poppel H, Wolff JM, Zattoni F, EAU Working Group on Oncological Urology: EAU guidelines on prostate cancer. Eur Urol 2001;40:97101.

11 Parker CC, Husband J, Dearnaley DP: Lymph node staging in clinically localized prostate cancer. Prost Cancer Prost Dis 1999;2:191199.

12 Wolf J, Cher M, Dall'Era M, Presti JC, Hricak H, Cavon PR: The use and accuracy of crosssectional imaging and fine needle aspiration cytology for detection of pelvic lymph node metastases before radical prostatectomy. J Urol 1995;153:993-999. 
13 Polascik TJ, Manyak MJ, Haseman MK, Gurganus RT, Rogers B, Maguire RT, Partin AW Comparison of clinical staging algorithms and 111-indium-capromab pendetide immunoscintigraphy in the prediction of lymph node involvement in high risk prostate carcinoma patients. Cancer 1999;85:1586-1592.

14 Rigo P, Paulus BJ, Kaschten BJ, Hustinx R, Bury T, Jerusalem G, Benoit T, Foidart-Willems J: Oncological applications of positron emission tomography with fluorine-18 fluorodeoxyglucose. Eur J Nucl Med 1996;23:16411674.

15 Wilkinson BA, Hamdy FC: State-of-the-art in prostate cancer. BJU Int 2001;87:423-430.

1616 Paul DB, Loening SA, Narayana AS, Culp DA: Morbidity from pelvic lymphadenectomy in staging carcinoma of the prostate. J Uro 1983;129:1141-1144.

17 Schuessler WW, Pharand D, Vancaille TG: Laparoscopic standard pelvic node dissection for carcinoma of the prostate: Is it accurate? J Urol 1993;150:898-901.

18 Mc Dowell GC, Johnson JW, Tenney DM, Johnson DE: Pelvic lymphadenectomy for staging clinically localized prostate cancer: Indications, complications, and results in 217 cases. Urology 1990;35:476-482.

19 Golimbu M, Morales P, Al-Askari S, Brown J Extended pelvic lymphadenectomy for prostatic cancer. J Urol 1979;121:617-620.
20 Alazraki NP, Eshima D, Eshima LA, Herda SC, Murray DR, Vansant JP, Taylor AT: Lymphscintigraphy, the sentinel node concept, and the intraoperative gamma probe in melanoma, breast cancer, and other potential cancers. Semin Nucl Med 1997;27:55-67.

21 Horenblas S, Jansen L, Meinhardt W, Hoefnagel CA, De Jong D, Nieweg OE: Detection of occult metastasis in squamous cell carcinoma of the penis using a dynamic sentinel node procedure. J Urol 2000;163:100-104.

22 Wawroschek F, Vogt H, Bachter D, Weckermann D, Hamm M, Harzmann R: First experience of gamma probe guided sentinel lymph node surgery in penile cancer. Urol Res 2000; 28:246-249.

23 Morton DL, Wen DR, Cochran AJ: Management of early stage melanoma by intraoperative lymphatic mapping and selective lymphadenectomy: An alternative to routine elective lymphadenectomy or watch and wait. Surg Oncol Clin N Am 1990;1:247-259.

24 Kubik S: Anatomie des Lymphgefässystems; in Földi M, Kubik S (eds): Lehrbuch der Lymphologie. Stuttgart, Gustav Fischer, 1993, p 168.

25 Partin AW, Kattan MW, Subong ENP, Walsh PC, Wojno KJ, Oesterling JE, Scardino P, Pearson JD: Combination of prostate-specific antigen, clinical stage, and Gleason score to predict pathological stage of localized prostate cancer. JAMA 1997;277:1445-1451.
26 Stone NN, Stock RG, Unger P: Laparoscopic pelvic lymph node dissection for prostate cancer: Comparison of the extended and modified techniques. J Urol 1997;158:1891-1894.

27 Lezin MS, Cherrie R, Cattolica E: Comparison of laparoscopic and minilaparotomy pelvic lymphadenectomy for prostate cancer staging in a community practice. Urology 1997;49:60 64

28 Fahlenkamp D, Müller W, Schönberger B, Loening SA: Laparoskopische pelvine Lymphadenektomie (LPLA) in der Diagnostik des lokoregionären Prostatakarzinoms. Akt Urol 1997;28:35-42.

29 Herrell SD, Trachtenberg J, Theodorescu D: Staging pelvic lymphadenectomy for localized carcinoma of the prostate: A comparison of 3 surgical techniques. J Urol 1997;157:13371339.

30 Wawroschek F, Wagner Th, Hamm M, Weckermann D, Vogt H, Märkl B, Gordijn R, Harzmann R: The influence of serial sections, immunohistochemistry, and extension of pelvic lymph node dissection on the lymph node status in clinically localized prostate cancer. Eur Urol, in press. 